\title{
АВТОМАТИЗИРОВАННЫЙ АЛГОРИТМ СИСТЕМНОГО АНАЛИЗА КОНКУРЕНТОСПОСОБНОСТИ ЦИФРОВОГО ПРЕДПРИЯТИЯ В РАМКАХ ИНФОРМАЦИОННОЙ СРЕДЫ
}

\author{
(c) 2021 Родионов Дмитрий Григорьевич \\ доктор экономических наук, профессор, Высшая инженерно-экономическая школа \\ Санкт-Петербургский политехнический университет Петра Великого, Россия, Санкт-Петербург \\ E-mail: dmitry.rodionov@spbstu.ru
}

(c) 2021 Мугутдинов Рашид Магомедрасулович

соискатель, Высшая инженерно-экономическая школа

Санкт-Петербургский политехнический университет Петра Великого, Россия, Санкт-Петербург

(C) 2021 Конников Евгений Александрович

кандидат экономических наук, доцент, Высшая инженерно-экономическая школа

Санкт-Петербургский политехнический университет Петра Великого, Россия, Санкт-Петербург

E-mail: konnikov.evgeniy@gmail.com

Процесс становления цифровой экономики на данный момент является объективной реальностью. Данный процесс в первую очередь характеризуется двумя базовыми системно связанными процессами - глобализации и цифровизации. Одним из ключевых следствий данного процесса является трансформация традиционного предприятия в цифровое предприятие. Цифровое предприятие для потребителя существует исключительно в рамках цифровой информационной среды, что в значительной мере ограничивает возможности маркетингового взаимодействия. Таким образом, цифровое предприятие является принципиально новым субъектом экономики, значительно отличающимся от традиционного предприятия. Авторы в рамках статьи [1] проанализировали свойства формирования конкурентоспособности цифрового предприятия и разработали адаптивный инструмент системного анализа конкурентоспособности цифрового предприятия в рамках информационной среды. В рамках данной статьи приводятся результаты алгоритмизации и автоматизации средствами высокоуровневого языка программирования Pуthon разработанного ранее инструментария.

Ключевые слова: цифровое предприятия, информационная среда, информационный срез, конкурентоспособность, тональность, системный анализ

Процесс цифровизации экономики на данный момент привел к появлению принципиально нового типа предприятия - цифрового предприятия. Цифровое предприятие для потребителя существует исключительно в рамках цифровой информационной среды, что в значительной мере ограничивает возможности маркетингового взаимодействия. Таким образом, цифровое предприятие является принципиально новым субъектом экономики, значительно отличающимся от традиционного предприятия. Ранее авторами был разработан и представлен в статье [1] инструментарий системного анализа конкурентоспособности цифрового предприятия в рамках информационной среды, который сущностно представляет собой совокупность порядковых метрик, многомерное восприятие ко- торых позволяет формулировать комплексные выводы относительно содержания и вектора развития образа предприятия в информационной среде. При этом приведённая совокупность метрик предполагает непрерывный мониторинг, обработку и квантификацию значительных объемов естественной цифровой информации. Следовательно использование данных метрик в рамках прикладной деятельности предприятия предполагает алгоритмизацию процесса их калькуляции и автоматизацию разработанных алгоритмов.

В первую очередь необходимо алгоритмизировать процесс поиска и агрегирования базой информации, описывающей образ предприятия в информационной среде. Данная информация значительно дифференцирована как с точки 
зрения источника, так и точки зрения потенциальной значимости. Для целей данного исследования можно выделить следующие основные информационные совокупности:

1. Информация, генерируемая непосредственно исследуемым предприятием и размещаемая им в рамках управляемых цифровых ресурсов, таких как официальный сайт и страницы предприятия в социальных сетях. Данный информационный поток является полностью управляемым, однако именно осознание данного факта снижает его содержательную значимость для потенциального потребителя. Более того, данный информационный поток крайне дифференцирован с динамической точки зрения, что значительно снижает значимость выводов при его конкурентном сопоставлении.

2. Информация, генерируемая потребителями ТРу предприятия в рамках специализированных ресурсов. Данная информация более значима для потенциального потребителя, так как является исключительно ограниченно управляемой исследуемым предприятием. Однако поиск и потребление данной информации является для потенциального потребителя трудоемким процессом. Более того, потребитель предварительно должен исследовать данные специализированные информационные ресурсы. Таким образом анализ данного информационного потока целесообразен исключительно при сравнении крайне масштабных в рамках информационной среды предприятий.

3. Информация условно стохастического характера, агрегируемая поисковыми ресурсами в рамках базового запроса. Описываемый информационный поток объединяет как части вышеперечисленной информации, так и иный информационные проявления, идентифицированные алгоритмами поисковых систем, таких как Яндекс или Гугл. Именно данная информация как правило формирует у потенциально- го потребителя первичное восприятие образа предприятия и становится базисом для принятия решения о взаимодействии с предприятием.

Таким образом именно информация условно стохастического характера является базисом конкурентного сопоставления. Алгоритмически, процесс агрегирования данной информации подразумевает формирование первичного запроса. Первичным запросом как правило является краткое содержательное обращение к поисковой системе. В рамках описываемого инструментария первичным запросом может выступать наименование предприятия. Алгоритмы существующих поисковых систем являются крайне комплексными и неуклонно совершенствуются, а результатом их работы выступает совокупность информационных ресурсов, описывающих содержание первичного запроса. Помимо ссылок на данные информационные ресурсы формируемый поисковой системой отчет содержит краткую информацию о содержании данного информационного ресурса. Именно данное содержание и выступает базисом формирования образа предприятия в сознании потенциального потребителя. Укрупненный алгоритм агрегирования данной информации представлен на рисунке 1 .

Представленный алгоритм требует автоматизации. Описанный процесс специалистами в области анализа данных именуется «Парсинг». Для целей автоматизированного парсинга как привило используются готовые решения, агрегированные в форме инструментальных библиотек на языке программирования Python. Данная инструментальная специфика обусловлена высоким уровнем универсальности и доступности языка программирования Python. Однако существующие поисковые системы как правило не одобряют процесс парсинга, следствием чего является необходимость моделирования поведения человека, для целей обхода сформи-
Эman I

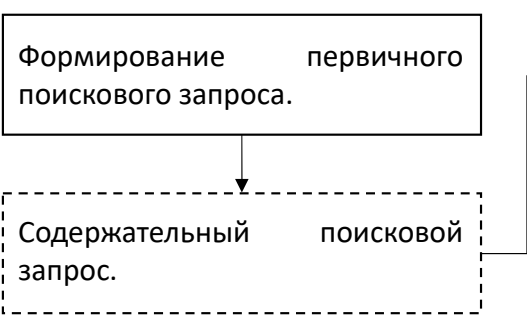

Этап II

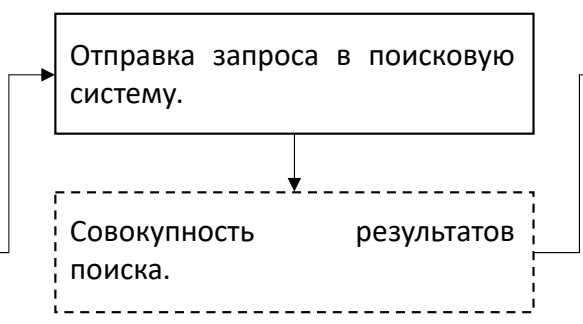

Эman III

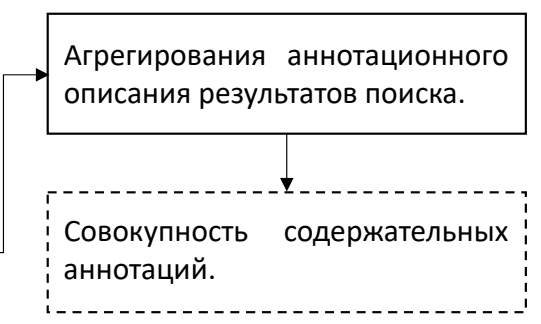

Рисунок 1. Алгоритм поиска и агрегирования базовой информации 
рованных поисковыми системами ограничений. Основным инструментом моделирования поведения человека в сети интернет является Webdriver библиотеки Selenium. В качестве поисковой системы наиболее целесообразно использовать Яндекс, так как системы защиты от парсинга у данной поисковой системы наиболее традиционные и наименее трудоемкие с точки зрения преодоления. Автоматизированный алгоритм принимает на вход первичный поисковой запрос и количество страниц результатов поиска, которые необходимо обработать. Необходимо отметить, что количество страниц результатов поиска является крайне вариативным параметром и в первую очередь оно зависит от масштаба исследуемого предприятия в информационной среде. Практика применения данного алгоритма показала, что наиболее универсальным значением данного параметра является диапазон от 5 до 15 страниц. Точное значение определяется экспертно в зависимости от упомянутого ранее масштаба предприятия в информационной среде. Так как получаемые результаты содержат множество контент-элементов, необходимо идентифицировать теги, в которых содержится исключительно анализируемая аннотационная информация. Таким образом представленный алгоритм может быть заключен в цикл. Пороговым значением счетчика цикла выступает определённое экспертно количество страниц результатов поиска. Тело цикла состоит из последовательного извлечения полученного контента, извлечения и агрегирования анализируемой аннотационной информации и перехода на следующую страницу результатов поиска. Необходимо отметить, что интервалы перехода между страницами результатов поиска искусственно увеличены, так как данный прием позволяет обойти системы защиты от парсинга имитируя процесс визуального осмысления приведенного контента и принятия решения о переходе на следующую страница результатов поиска. Извлеченная аннотационная информация агрегируется в список, и представляет собой последовательность единиц естественной информации, представленной в текстовой форме. Именно данная последовательность является результатом применения алгоритма. На рисунке 2 представлена описанная автоматизация алгоритма поиска и агрегирования базовой информации.

Полученный по результатам применения данного алгоритма массив текстовой информации не пригоден для автоматизированного анализа, так как представлен исключительно в естественной форме, подразумевающей осмысление человеческим сознанием. Для целей автоматизированной обработки процесса сопоставления данный информационный массив необходимо представить в виде элементарных текстовых единиц - лексем или токенов.

Процесс токенизации подразумевает последовательное разделение текстовой информации на элементарные единицы и их последующую обработку. В рамках обработки первично извлеченных токенов в первую очередь реализуется лемматизация, т.е. переход к базовой форме токена. Полученный массив лемматизированных токенов в свою очередь подлежит содержательной фильтрации, подразумевающей отказ от низкосодержательных токенов, к которым в первую очередь относятся союзы, предлоги, местоимения и т.д. По результатам фильтрации формируется массив содержательных токенов, представляющих собой совокупность элементарных единиц формирования образа исследуемого предприятия в рамках информационной среды. Именно данные токены сущностно определяют специфику восприятия предприятия потенциальными потребителями, а следовательно, и его относительную конкурентоспособность. На рисунке 3 представлен алгоритм токенизации базовой информации.

В соответствии с приведенным алгоритмом можно заключить, что подобная обработка текстовой информации подразумевает использование потенциально комплексного инструментария. Необходимая совокупность инструментов автоматизирована в рамках библиотеки NLTK. На рисунке 4 представлена автоматизация данного алгоритма с использованием инструментов библиотеки NLTK.

Полученный массив токенов в первую очередь можно охарактеризовать спецификой частотного распределения. Типовым частотным распределением токенов в рамках массива естественной текстовой информации является распределение Ципфа. Данное распределение характеризуется экспоненциальным приростом частоты встречаемости токена при увеличении его порядкового номера в иерархически упорядоченном относительно частоты встречаемости списке. 


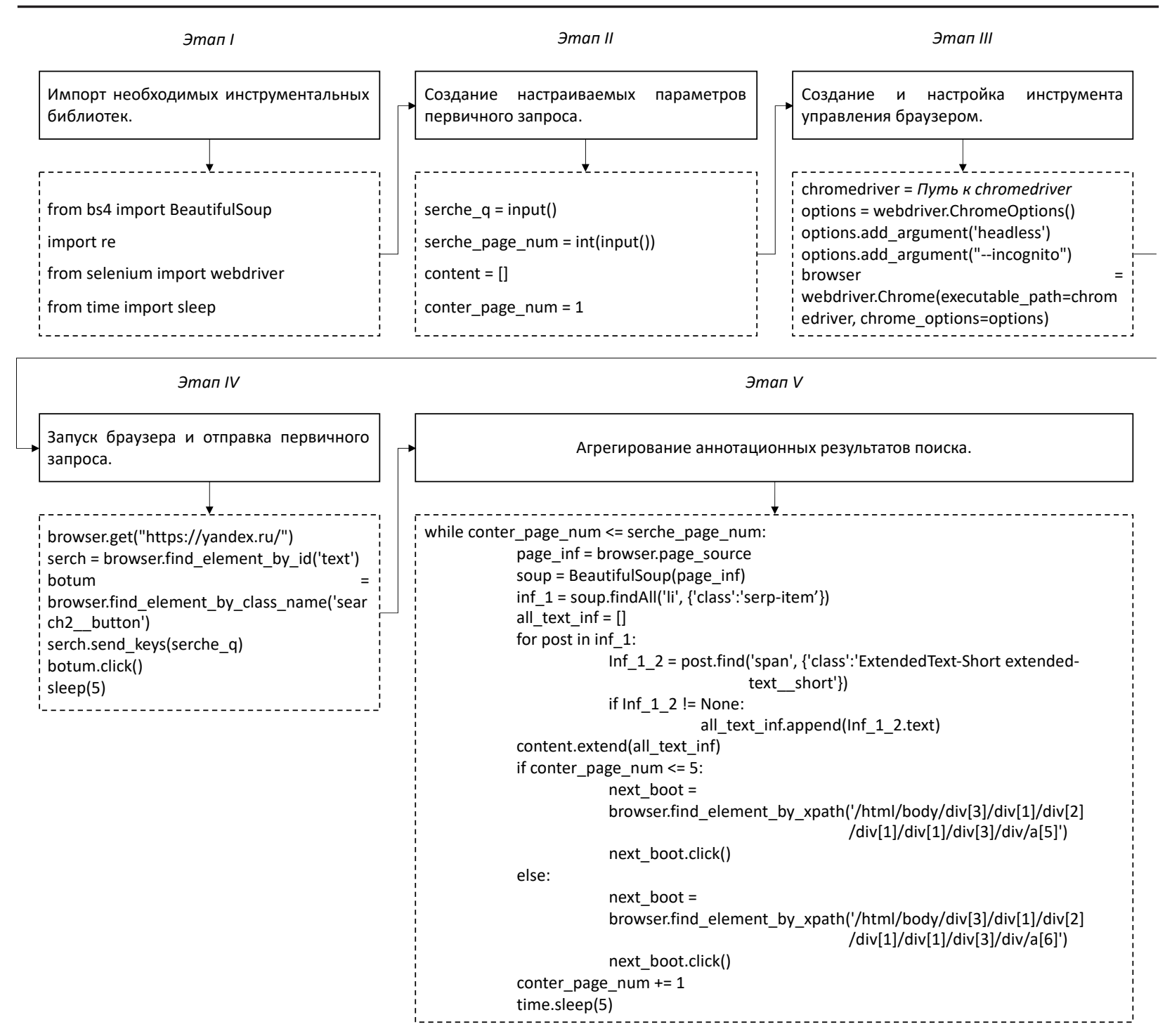

Рисунок 2. Автоматизация алгоритма поиска и агрегирования базовой информации

Эman I

\begin{tabular}{|l|}
\hline $\begin{array}{l}\text { Первичная } \\
\text { массива } \\
\text { информации. }\end{array}$ \\
\hline
\end{tabular}

Эman II

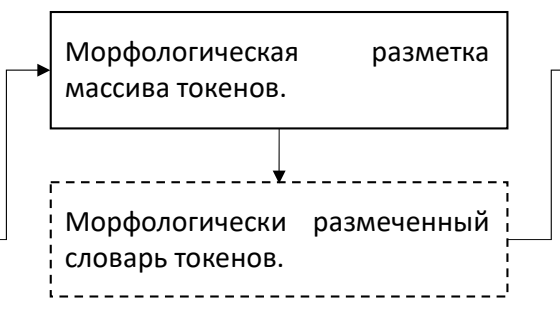

Эman III

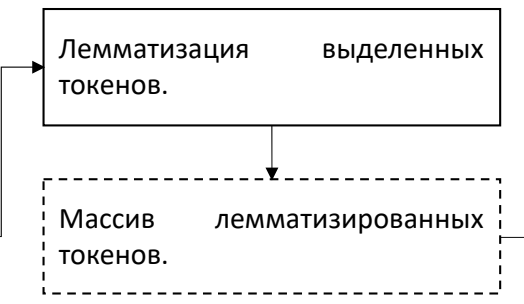

Эman IV

Исключение

низкосодержательных токенов.

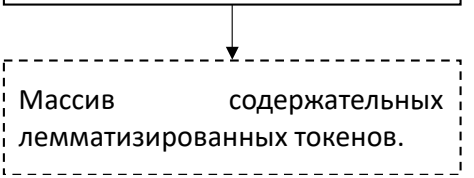




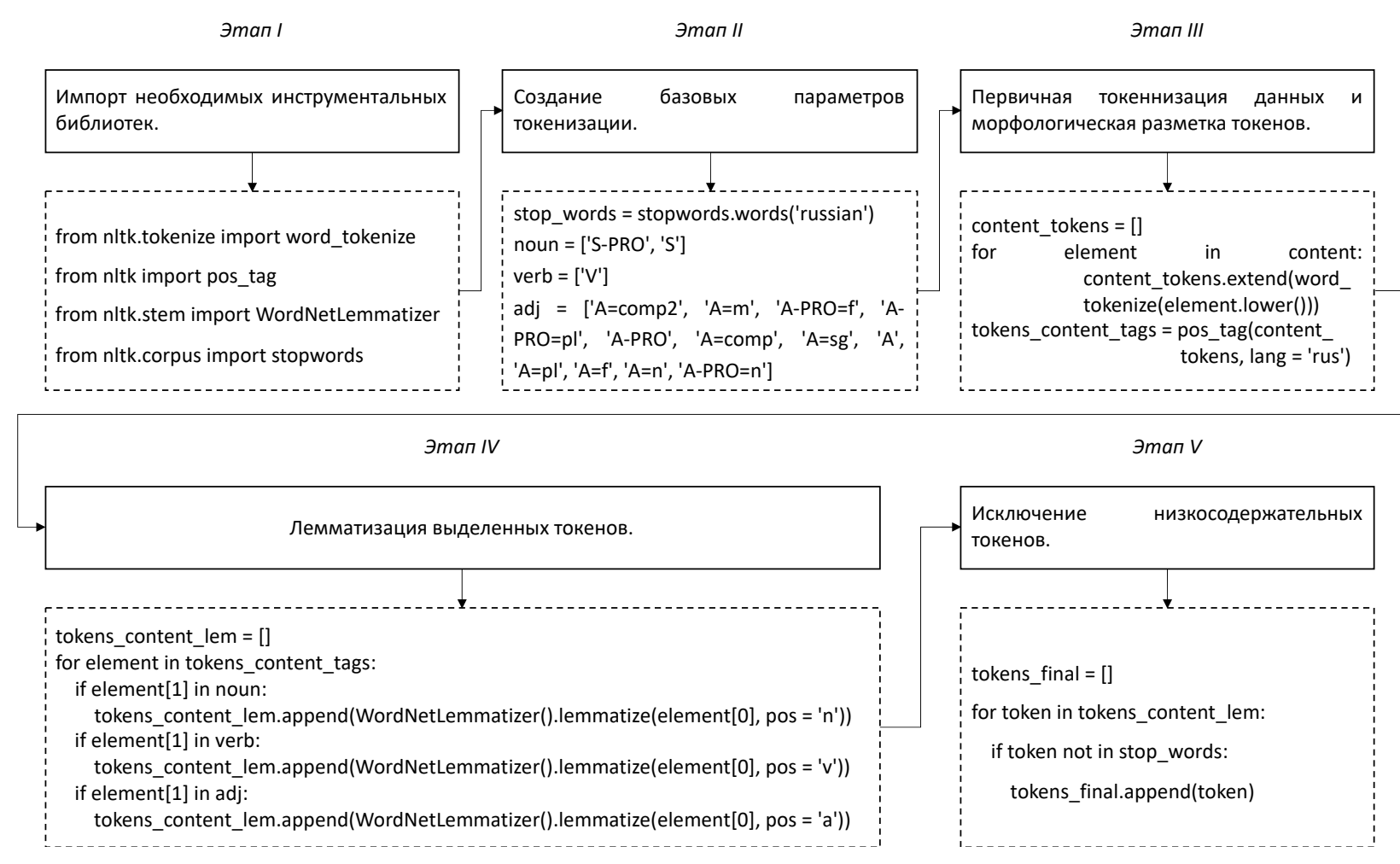

Рисунок 4. Автоматизация алгоритма токенизации базовой информации

Таким образом можно выделить значительный массив токенов, частота упоминания которых крайне мала. На рисунке 5 представлен пример распределения Ципфа.

Как можно видеть данное распределение позволяет разделить единый массив токенов на три сектора:

- Сектор I - наименее часто упоминаемые токены. Как правило в данный сектор входят токены, упомянутые не более одного раза. Данный массив токенов объединяет в себе наименее значимые с аналитической точки зрения токены. Следовательно, для целей повышения содержательно-аналитической значимости сформированного массива токенов данную совокупность необходимо исключить.

- Сектор II - токены «частотного перехода». Данная совокупность сравнительно меньше, чем совокупность токенов в секторе I, и как правило они представляют наибольшую аналитическую значимость, так как именно они являются уточняющими по отношению к наиболее часто встречающимся токенам.

- Сектор III - токены, являющиеся содержательными центроидами анализируемого текстового массива. Данные токены встречаются практически в каждой из единиц изначального текстового массива.
В рамках разрабатываемого инструментария необходимо предполагается выделение наиболее содержательно значимых, для формирования образа предприятия в сознании потенциальных потребителей, токенов. Следовательно, необходимо обработка исключительно токенов, содержащихся в секторе II секторе III. На рисунке 6 представлен алгоритм квантификации и фильтрации массива токенов.

Для целей автоматизации квантификации в рамках данного алгоритма может быть использован инструмент Counter библиотеки Collections. Эмпирически было определено, что универсальным в данном случае может выступать принцип отбора на основе 2 средних значений частоты. На рисунке 7 представлена автоматизация алгоритма квантификации и фильтрации массива токенов.

Сформированный массив токенов является содержательно достаточным для целей анализа образа исследуемого предприятия в рамках информационной среды. Однако с количественной точки зрения, сформированный массив не является достаточным. Получаемая совокупность токенов является содержательно однозначной, однако она представляет собой только часть потенциального массива, описывающего образ предприятия. Лексическое многообразие информационной среды определяет наличие 


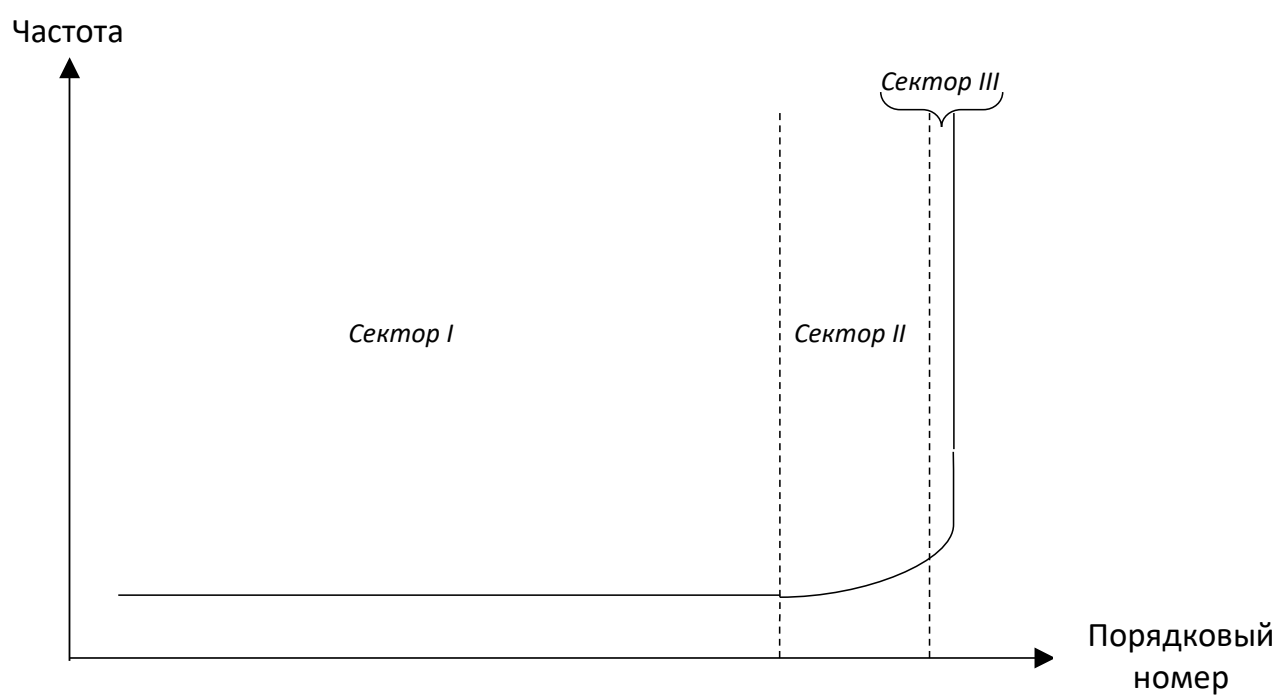

Рисунок 5. Распределение Ципфа

Этап I

Этап II

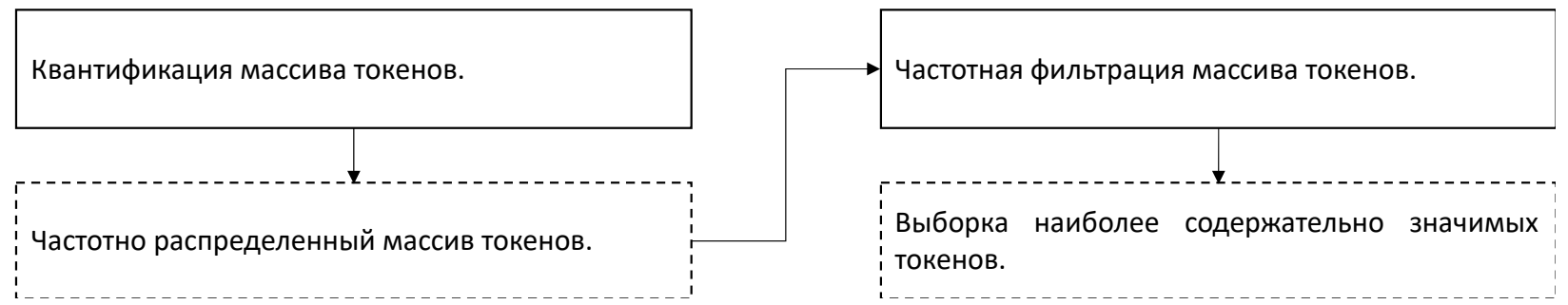

Рисунок 6. Алгоритм квантификации и фильтрации массива токенов

Этап I

Импорт необходимых инструментальных библиотек.

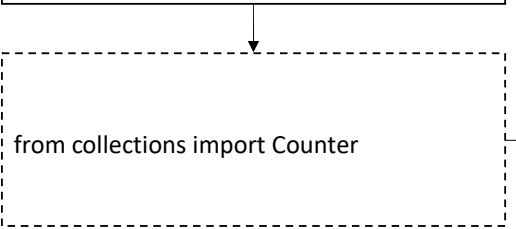

Эman II

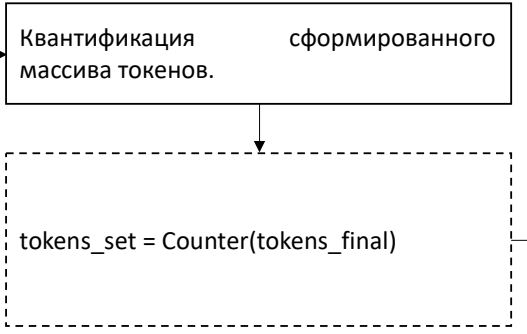

Эman III

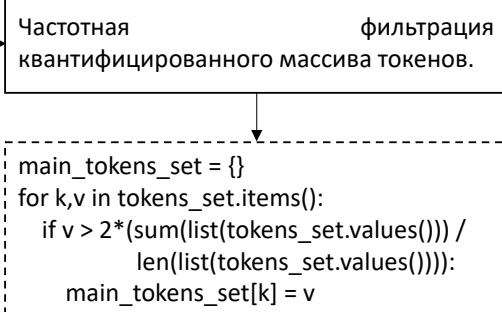

Рисунок 7. Автоматизация алгоритма квантификации и фильтрации массива токенов 
множества потенциально ассоциируемых с данным массивом токенов. Следовательно данный массив необходимо искусственно расширить за счет добавления ассоциативных токенов. Для данных целей может быть использован ресурс «https://wordassociations.net/». Данный ресурс позволяет сформировать массив ассоциативных токенов на основе базового токена. Алгоритм данной трансформации массива токенов представлен на рисунке 8.

Автоматизация данного алгоритма возможна посредствам использования инструмента Webdriver библиотеки Selenium. Данный инструмент позволяет сформировать адресных запрос к pecypcy «https://wordassociations.net/» и агрегировать получаемую для каждого токена совокупность ассоциативных токенов.

Для целей квантификации сформированного массива токенов также может быть использован описанный ранее инструмент Counter библиотеки Collections. Получаемая квантифицированная совокупность токенов также обладает свойствами распределения Ципфа, что определяет необходимость исключения наименее (в соответствии с частотой упоминания) содержательно значимых токенов. Автоматизация алгоритма ассоциативной трансформации массива токенов представлена на рисунке 9.
Эman I

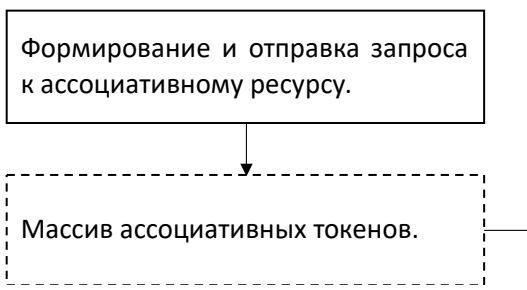

Эman II

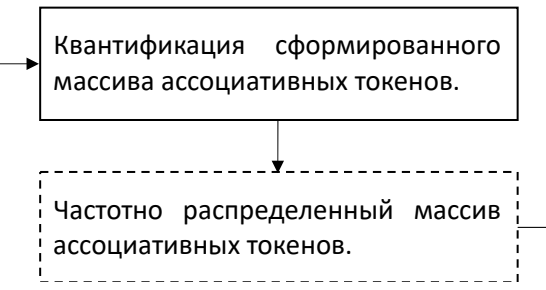

Эman III

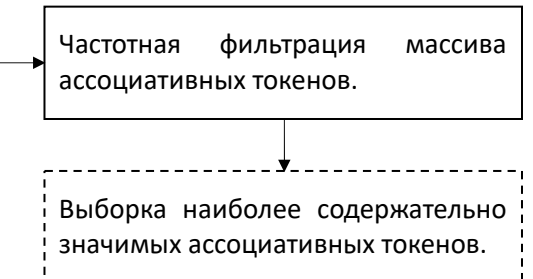

Рисунок 8. Алгоритм ассоциативной трансформации массива токенов

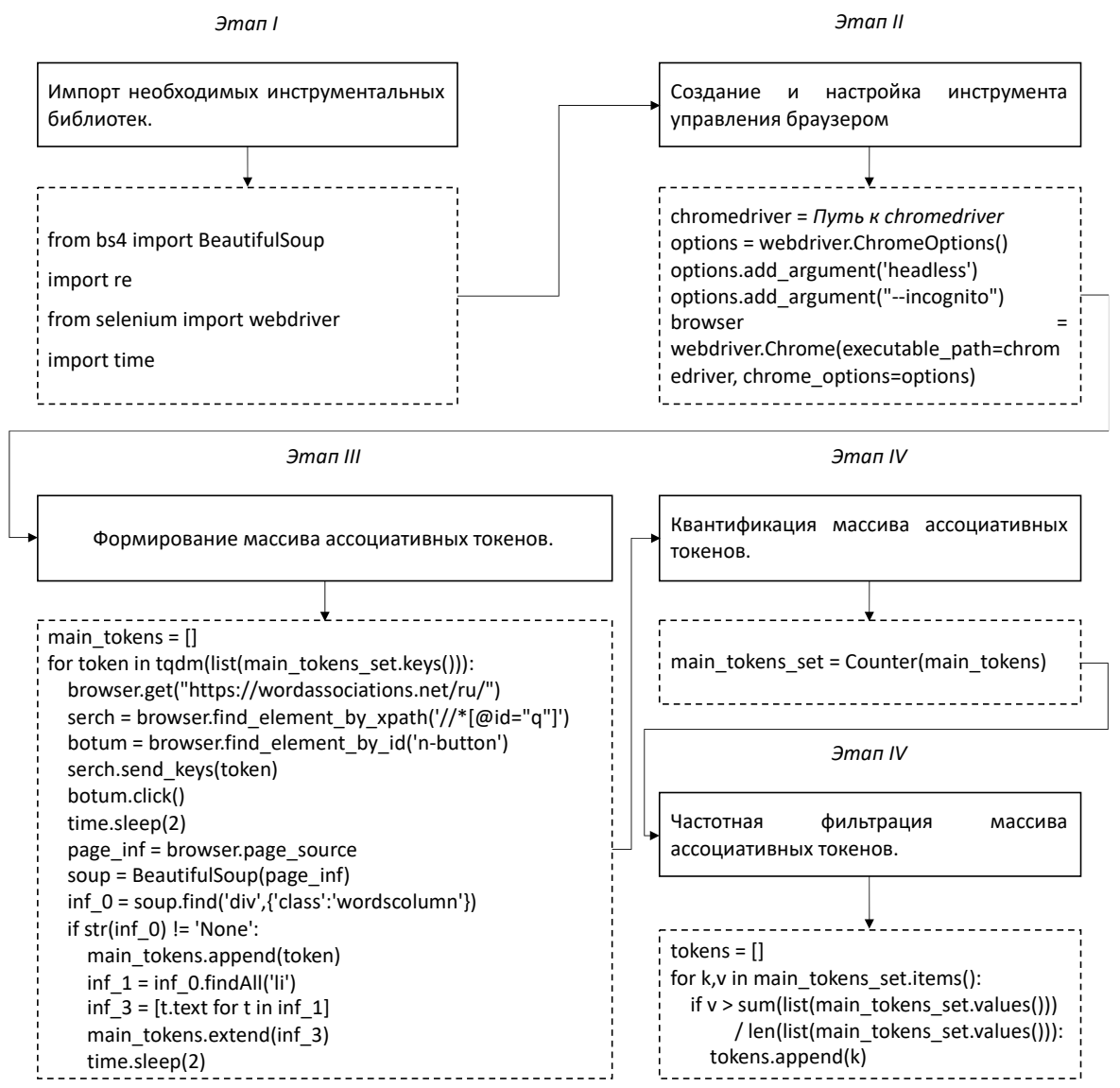


Результатом применения данного автоматизированного алгоритма является завершённый массив токенов, описывающих образ исследуемого предприятия в информационной среде. Для целей оценки содержательной конкурентоспособности необходимо определить долю в данном массиве токенов, описывающих ключевые конкурентные свойства данного предприятия. Ранее отмечалось, что в зависимости от отраслевой принадлежности или иной специфики ТРУ, данными токенами могут описываться такие свойства как функциональность, надежность, доступность и множество иных свойств. Для целей формирования массива токенов, описывающих ключевые конкурентные свойства предприятия может быть использован модифицированный алгоритм ассоциативной трансформации. Первичным этапом данного алгоритма является экспертное формирование основного массива токенов конкурентных свойств. Далее каждый из данных экспертно определенных токенов направляется на описанный ранее ресурс поиска ассоциативных токенов.

Получаемые массива ассоциативных токенов агрегируется в единый массив ассоциативных токенов конкурентных свойств. Алгоритм формирования массива токенов конкурентных свойств представлен на рисунке 10.

Следует отметить, что этап III и этап IV является вариативными, и в случае крайне малого объема первичного массива токенов конкурентных свойств, они могут быть исключены. На рисунке 11 представлена автоматизация упрощен- ного алгоритма формирования массива токенов конкурентных свойств.

Сформированные массивы токенов являются базисом определения разработанного коэффициента содержательной конкурентоспособности. Сопоставление данных массивов необходимо проводить с учетом нечетного сопоставления токенов. Для целей нечеткого сопоставления токенов наиболее эффективным инструментом является расстояние Левенштейна. В том случае если расстояние Левенштейна не ниже допустимого минимума, токен признается общим для обоих массивов. Допустимые значения расстояния Левенштейна крайне дифференцированы и могут варьироваться в зависимости от задачи. В данном случае в качестве допустимого значения данного параметра выбран порог в 80\%. На рисунке 12 представлен алгоритм определения коэффициента содержательной конкурентоспособности.

Для целей автоматизации данного алгоритма может быть использована библиотека Fuzzywuxxy, содержащая множество вариаций определения расстояния Левенштейна. На рисунке 13 представлена автоматизация данного алгоритма.

Получаемый результат необходимо сопоставить с нормированным значениями основных конкурентов, для чего может использоваться матрица информационной конкурентоспособности цифрового предприятия. Однако для целей многомерного сопоставления необходимо алгоритмизировать и автоматизировать про-
Эman I

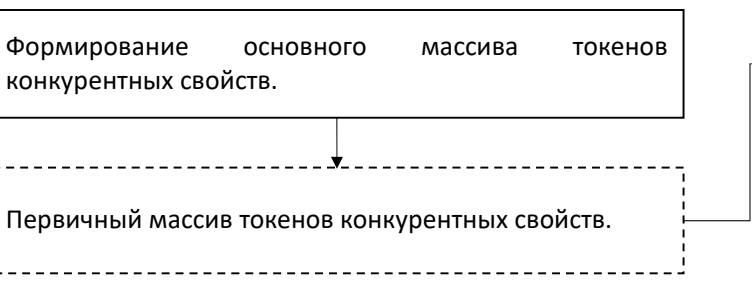

Эman II

Формирование и отправка запроса к ассоциативному ресурсу.

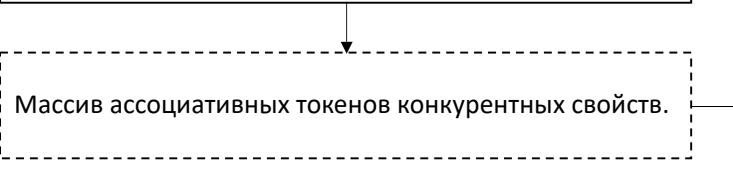

$\exists \operatorname{man} I V$

Частотная фильтрация массива ассоциативных токенов конкурентных свойств.

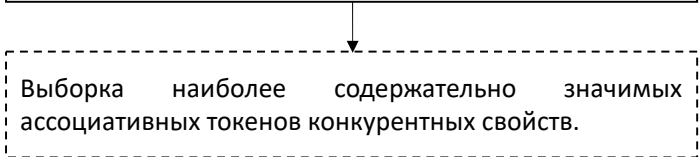




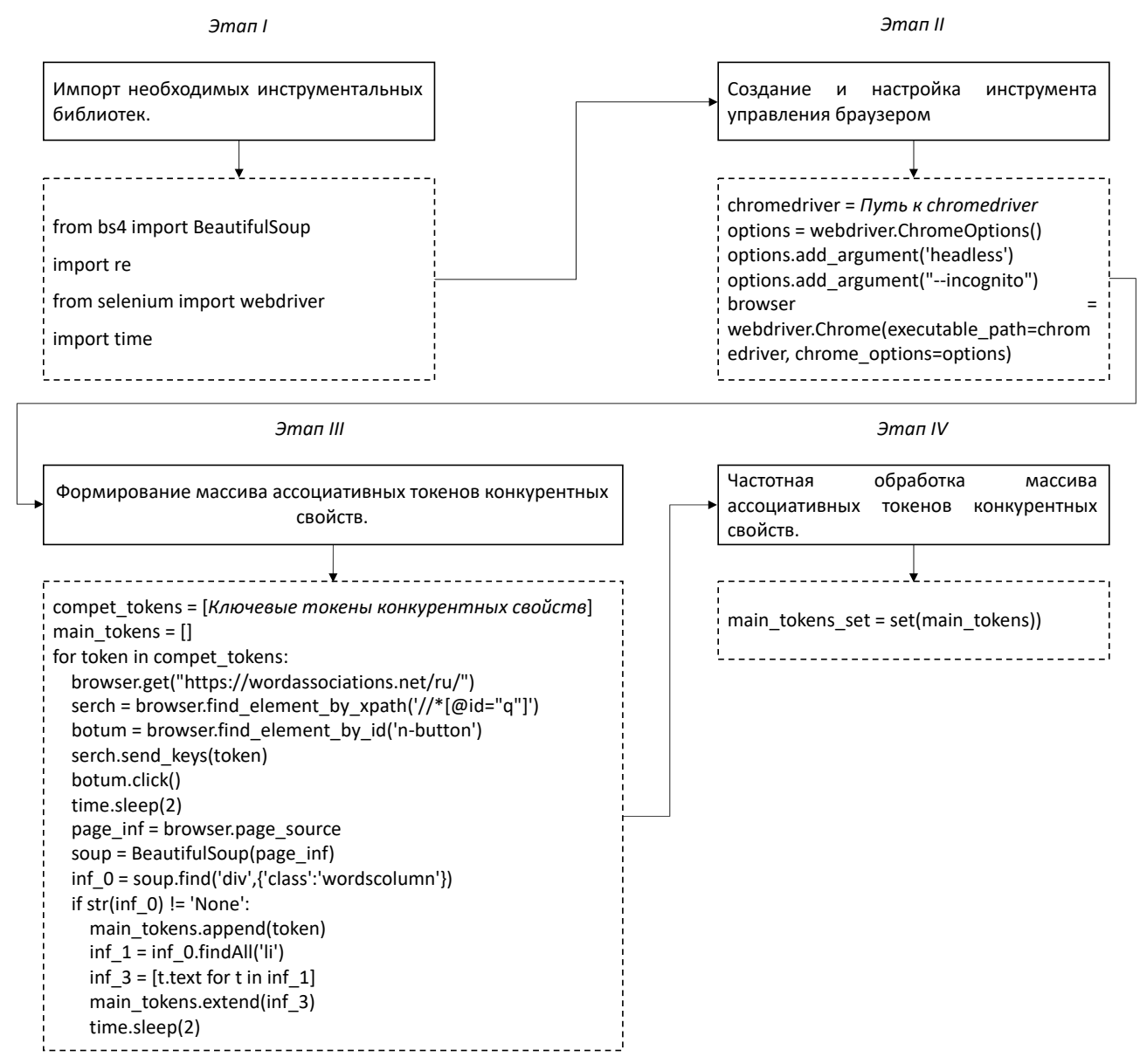

Рисунок 11. Автоматизация упрощенного алгоритма формирования массива токенов конкурентных свойств

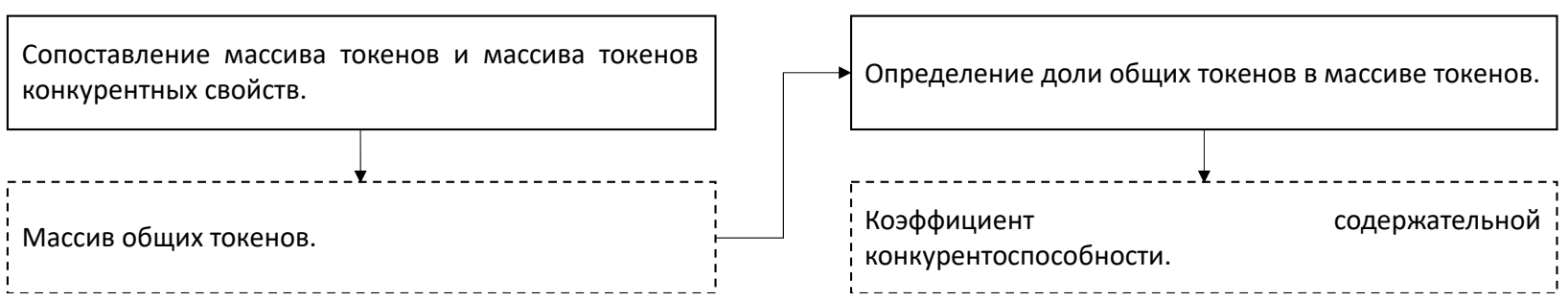

Рисунок 12. Алгоритм определения коэффициента содержательной конкурентоспособности

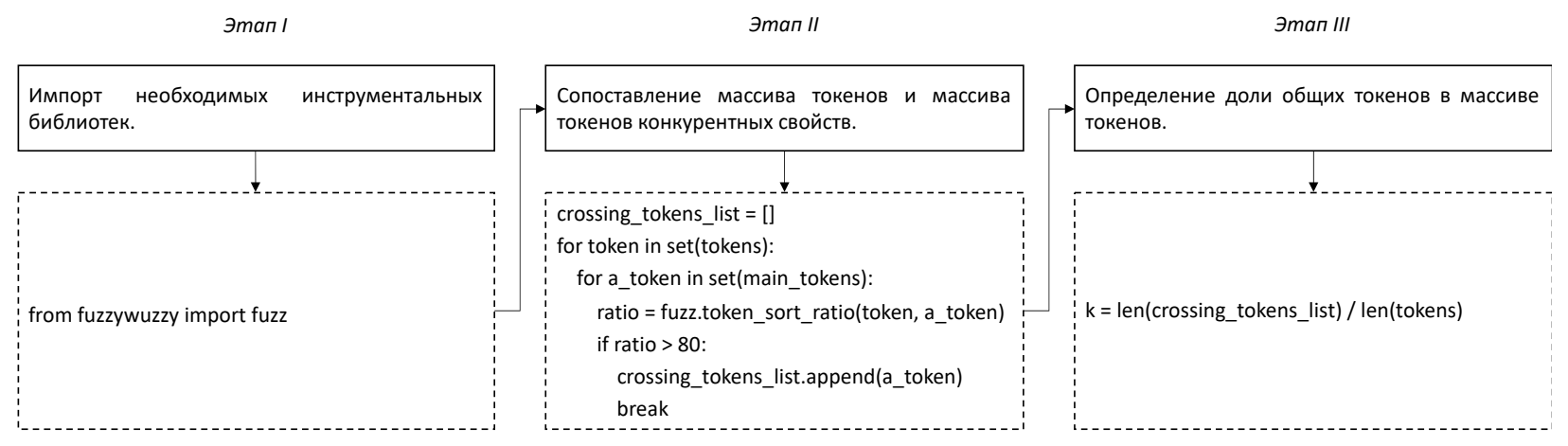

Рисунок 13. Автоматизация алгоритма определения коэффициента содержательной конкурентоспособности 
цесс калькуляции коэффициента тональной конкурентоспособности. В качестве базиса для расчета тональных характеристик эффективно использовать описанную ранее совокупность содержательных аннотаций.

На рисунке 14 представлен алгоритм определения коэффициента тональной конкурентоспособности.

Необходимо отметить, что так как сформированный массив содержательных аннотаций не обладает динамическими свойства и является одномерным с точки зрения времени, распре- деление удельного веса нецелесообразно. Для целей автоматизации данного алгоритма предлагается использовать библиотеку Dostoevsky. На рисунке 15 представлена автоматизация алгоритма определения коэффициента тональной конкурентоспособности.

Полученные результаты относительно исследуемого предприятия и его конкурентов формируют матрицу информационной конкурентоспособности цифрового предприятия, на основе анализа которой формулируются рекомендации к принятию управленческих решений.
Этап I

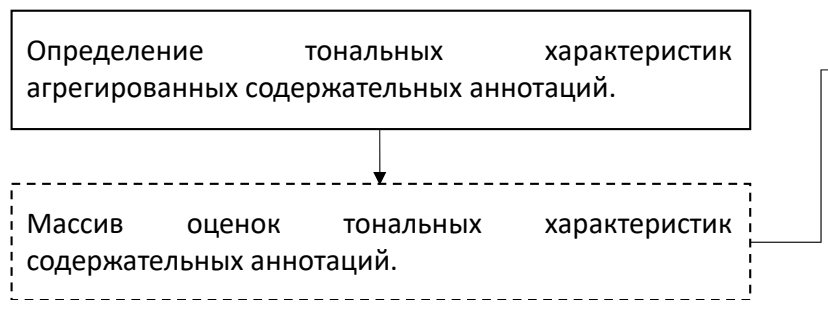

Этап II

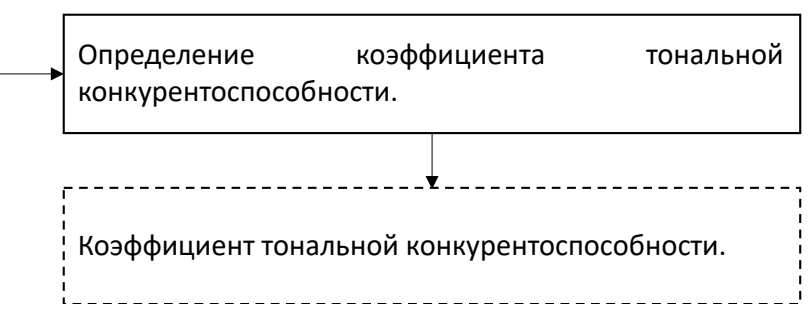

Рисунок 14. Алгоритм определения коэффициента тональной конкурентоспособности

Эman I

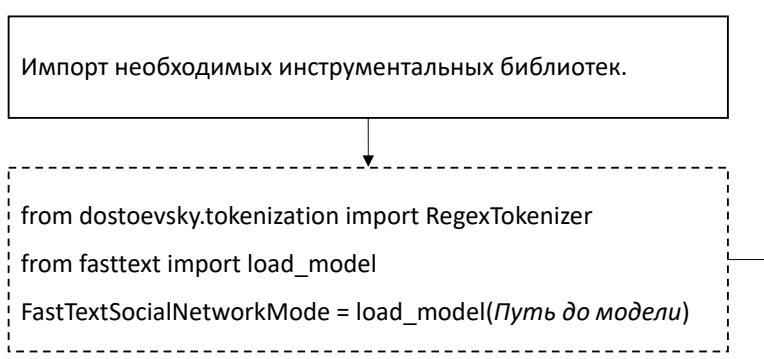

Эman II

Формирование базовых компонент.

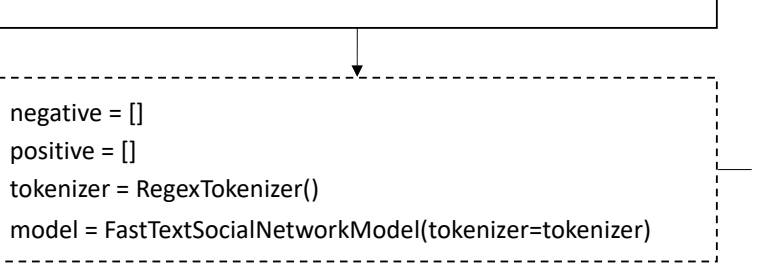

\begin{tabular}{|c|c|}
\hline Этап III & Этап IV \\
\hline $\begin{array}{l}\text { Определение уровня позитивности и негативности } \\
\text { содержательных аннотаций. }\end{array}$ & $\begin{array}{l}\text { Определение коэффициента тональной } \\
\text { конкурентоспособности. }\end{array}$ \\
\hline $\begin{array}{l}\text { for element in content: } \\
\text { tonal_analis = model.predict([element]) } \\
\text { for dictionary in tonal_analis: } \\
\text { for } k, v \text { in dictionary.items(): } \\
\text { if } k==\text { 'negative': } \\
\text { negative.append(v) } \\
\text { elif } k==\text { 'positive': } \\
\text { positive.append( } v)\end{array}$ & $\begin{array}{l}\text { k_list }=[] \\
\text { positive_counter = } 0 \\
\text { for element in negative: } \\
\text { k_list.append(positive[positive_counter] / element) } \\
\text { positive_counter }+=1 \\
\text { = sum (k_list)/len (k_list) }\end{array}$ \\
\hline
\end{tabular}

Рисунок 15. Автоматизация алгоритма определения коэффициента тональной конкурентоспособности 


\section{Библиографический список}

1. Родионов, Д.Г. Системный анализ конкурентоспособности цифрового предприятия в рамках информационной среды / Д. Г. Родионов, Е.А. Конников, Р. М. Мугутдинов // Экономические науки. - 2020.- № 193.- С. 394-401.- DOI 10.14451/1.193.394.

2. Родионов, Д. Г. Методология системного анализа информационной среды / Д. Г. Родионов, Е.А.Конников, О.А. Конникова // Экономические науки. - 2021._- № 196.- С. 160-174._- DOI 10.14451/1.196.160.

3. Родионов, Д. Г. Информационный капитал предприятия как целевой показатель развития в рамках цифровых экономических систем / Д. Г. Родионов, Е. А. Конников, Д. А. Алферьев // Экономические науки. - 2020.№ 190.- С. 131-137.- DOI 10.14451/1.190.131.

4. Родионов, Д.Г. Влияние информационной среды на субъекты малого и среднего предпринимательства / Д.Г. Родионов, С.В.Ялымов, Е.А.Конников // Экономические науки. - 2020. - № 189.- C. 86-91.- DOI 10.14451/1.189.86.

5. Мазилкина Е.И., Паничкина Г.Г. Управление конкурентоспособностью Издательство: Омега-Л Год: 2009 , С. 27.

6. Конников, Е. А. Маркетинг некропроизводств / Е.А. Конников, О.А. Погребова // Вестник факультета управления СПбГЭУ.-2017.- № 1-1.- С. 277-280.

7. Термин: Лемматизация.- Текст: электронный // Система PromoPult: [сайт].- URL: https://promopult.ru/ library/Лемматизация (дата обращения: 14.07.2021). 